\title{
In-Space Crew-Collaborative Task Scheduling
}

\author{
John Jaap 56806 \\ John Jaap," Patrick Meyer," Elizabeth Davis," and Lea Richardson* \\ National Aeronautics and Space Administration, Marshall Space Flight Center, Alabama, 35812
}

\begin{abstract}
As humans venture farther from earth for longer durations, it will become essential for those on the journey to have significant control over the scheduling of their own activities as well as the activities of their companion systems and robots. However, there are many reasons why the crew will not do all the scheduling; timelines will be the result of collaboration with ground personnel. Emerging technologies such as in-space message buses, delay-tolerant networks, and in-space internet will be the carriers on which the collaboration rides. Advances in scheduling technology, in the areas of task modeling, scheduling engines, and user interfaces will allow the crew to become virtual scheduling experts. New concepts of operations for producing the timeline will allow the crew and the ground support to collaborate while providing safeguards to ensure that the mission will be effectively accomplished without endangering the systems or personnel.
\end{abstract}

\section{Introduction}

For OR all past and current human space missions, the final scheduling of tasks to be done in space has been devoid of crew control, flexibility, and insight. Ground controllers, with minimal input from the crew, schedule the tasks and uplink the timeline to the crew or uplink the command sequences to the hardware. Prior to the International Space Station (ISS), the crew could make requests about tomorrow's timeline, they could omit a task, or they could request that something in the timeline be delayed. This lack of control over one's own schedule has had negative consequences (Ref. 1). There is anecdotal consensus among astronauts that control over their own schedules will mitigate the stresses of long-duration missions. On ISS, a modicum of crew control is provided by the "job jar." Ground controllers prepare a task list (a.k.a. "job jar") of non-conflicting tasks from which jobs can be chosen by the in-space crew. Because there is little free time and few interesting non-conflicting activities, the tasklist approach provides little relief from the tedium of being micro-managed by the timeline.

Scheduling for space missions is a complex and laborious undertaking which usually requires a large cadre of trained specialists and suites of complex software tools. It is a giant leap from today's ground-prepared timeline (with a job jar) to full crew control of their schedule. However, technological advances, currently in-work or proposed, make it reasonable to consider scheduling as a collaborative effort of the ground-based teams and the inspace crew. Collaboration would allow the crew to make minor adjustments, add tasks to suit their preferences, understand the reasons for the placement of tasks on the schedule, and provide them a sense of control over their own schedules. In foreseeable but extraordinary situations, such as quick response to anomalies and extended or unexpected loss of signal, the crew could have the autonomous ability to make appropriate modifications to the timeline, extend the timeline, or even start over with a new timeline.

The Vision for Space Exploration (VSE), currently being pursued by the National Aeronautics and Space Administration (NASA), will send humans to Mars in a few decades. Stresses on the human mind will be exacerbated by the longer durations and greater distances, and it will be imperative to implement stress-reducing innovations such as giving the crew control of their daily activities.

\section{A. Major Consideration}

Implementation of crew collaboration will be driven by one major circumstance - the round-trip light-time delay between the Earth and Mars can be up to 44 minutes and will never be less than 6 minutes (Fig. 1). Every 26 months, Mars cycles between close approach and farthest retreat. Barring some breakthrough in propulsion technologies, a mission to Mars will take longer than two years during which the light-time delays will average over 20 minutes. Normal voice conversations will be impossible. Instant transfer of information as provided by today's earth-based internet will also be negated. The communication delays will change the way human missions are

\footnotetext{
* Mission Planning Engineer, EO50.
} 
operated. The crew will be the first responders to emergencies and mundane anomalies; they will attend autonomously to all alarms, switching the troublesome system to a safe mode and/or making quick repairs and reconfigurations. What we now think of as ground control teams will become ground support teams.

The inability to have normal conversations with the ground, seeing the earth only as a point of light, having no immediate return to earth options, interacting only with their companions,

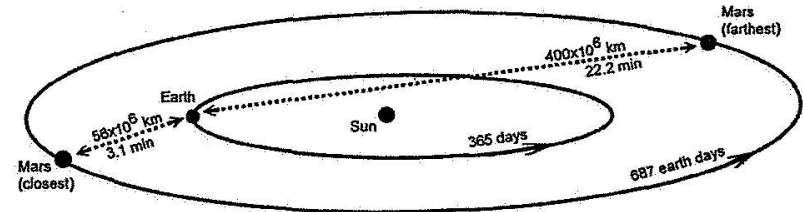

Figure 1. Light-Time Delays to Mars having limited food choices, drinking recycled water, and doing the same maintenance tasks each day will make life stressful for the crew. Crew collaboration on the development of the daily schedule for themselves and for the systems they use and maintain will provide them necessary knowledge to execute the plan and necessary influence so that they will feel that they are in control of their own destiny.

\section{B. Collaboration}

Collaboration is working jointly to produce a product or to attain a goal. Usually collaborators share ideas, compromise on goals or methods, contribute where their expertise allows, and jointly arrive at an objective. There are two types of collaboration, interactive and passive. Interactive implies that the collaborators communicate during the collaboration process. Passive collaboration happens without direct communication. An instance of interactive is a group of authors who brainstorm the contents of a paper. An instance of passive collaboration is the progression of teachers who instruct children from early childhood into adults; another instance is the corps of guards who attend all the gates at an arena so that only ticket holders are allowed to enter.

There are several methods which support interactive collaboration; often, these are used in combinations. The more common methods are listed below in approximate order of productivity.

- Face-to-face - collaborators talk to one another across the table, use whiteboards, share notes, etc.

- Teleconferencing and web conferencing - collaborators simulate being face-to-face using voice, possibly video, and sometimes an electronic white board or a shared computer "desktop."

- Custom software - an application designed to support collaboration on a specific task or product. Internet games are a common example of custom software.

- Instant messaging and chat rooms - collaborators type messages which are displayed almost instantly for each collaborator.

- File transfer - collaborators post and retrieve files using peer-to-peer transfer or a common drop box. Commercial products are available to automate this method.

- Electronic forums - collaborators post messages on a message board.

- Electronic mail - collaborators correspond by sending messages over the internet.

- Postal mail - collaborators correspond by written mail.

Passive collaboration is based on a concept of operations rather than communication.

For earth-based support teams and humans on a mission to Mars, several of these collaboration methods will not be available and others (file transfer, electronic forums, and electronic mail) will be degraded. Custom software and the concept of operations can be tailored to deal with the time delays. Collaboration between the in-space crew and the ground support will, no doubt, use all the tools available including a delay-tolerant communication infrastructure, delay-tolerant scheduling software, and a specially tailored concept of operations.

\section{Concept of Operations}

Human missions to the moon and Mars will use extraordinarily complex hardware and software systems and will include significant technological and scientific investigations. The missions will extend for years, and ground support will require collaboration from many widely dispersed contributors. The cost to collect the ground-based planning and scheduling collaborators in a central location will be prohibitive. The only affordable solution will be to distribute the planning and scheduling efforts. It logically follows that if the planning and scheduling effort is to be distributed, it can be distributed to the crew on the moon, in transit to Mars and at Mars. Distributed planning and scheduling has been used to a small extent for ISS. Research into concepts of operations (Ref. 2) which maximize 
the distribution and the cost savings has yielded viable results. ${ }^{\dagger}$ To be successfully accepted and implemented, a distributed concept of operations must be considered and developed beginning as early as possible. The concept proposed here is intended to be a starting point for the concept which will eventually be used to allow full distribution of the planning and scheduling function to all collaborators including the in-space crew. The concept has the additional advantage of giving the crew full autonomy if they should need it. Figure 2 shows the contributions to the planning and scheduling effort by different collaborators during the preparation of the preliminary plan on earth and after the plan is uplinked to the crew. Text messages, notes, email, and voice memos are not shown on the chart. The development of the schedule is divided into two phases, preliminary and final. Preliminary schedules are developed on the ground using a ground-based instance of the scheduling system. Before the beginning of each work day, the schedule is transferred to an in-space instance of the scheduling system. In-space crew has timedelayed access to the ground instance and the ground support has time-delayed access to the in-space instance.

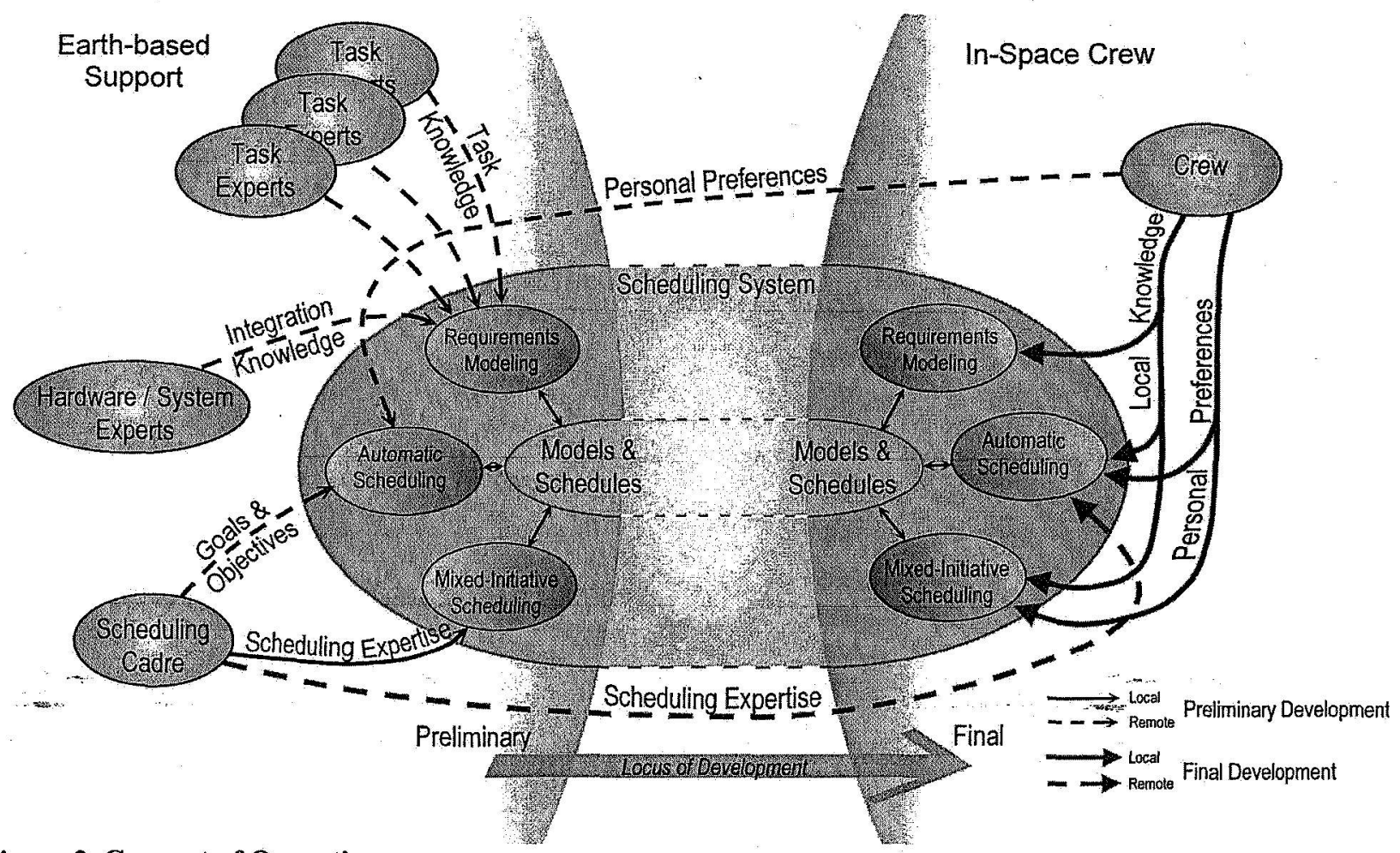

Figure 2. Concept of Operations

\section{A. Wide-Spread Collaboration on Preliminary Schedule}

Collaboration is cost effective because those with the best knowledge are able to contribute that knowledge directly. For the same reason, collaboration produces the best product. Collaboration on the planning and scheduling effort for the preliminary schedule is described by listing what each collaborator contributes.

- Task Experts - Task experts include hardware developers, scientists, engineering support teams, doctors, astrobiologists and others who have first-hand knowledge about the tasks to be done to maintain the vehicle/habitats and to conduct the science activities. These experts define the tasks and arrange them into the required sequences in order to accomplish the goals. The task definitions include the procedure descriptions, the equipment (and operation modes) to be used, the conditions required, and the durations. These task models do not directly define the resource amounts required by the tasks; resources are defined by the equipment mode models entered by the hardware and systems experts. The sequence definitions include the temporal relationships between the tasks, relationships to other

\footnotetext{
${ }^{\dagger}$ Fully distributed concepts of operations will not be implemented for $I S S$ because the paradigm shift would require rewriting current international agreements, incur appreciable one-time costs including new software, procedures, and training, and the phase over would extend almost to the end of the ISS mission.
} 
sequences, windows of opportunity and other data. The information is entered into a central data repository used by the scheduling system.

- Hardware and System Experts - Hardware and systems experts have detailed knowledge about how the hardware is integrated into the vehicle and how that hardware can be used. They also have knowledge about the software systems and how they behave. The experts enter the equipment mode models into the central data repository. For each mode of each piece of equipment, these models define how much of each resource is used. For example, an oven for metallurgical analysis of regolith samples has several operation modes using different resources (power, inert gases, data rates, etc.) and amounts. The hardware and systems experts know how the oven functions and how it is connected to the systems (which power bus, which controller, which video bus, etc.). These equipment mode models are used by the task experts when defining the tasks; therefore, these models eliminate the need for task experts to be hardware experts.

- Scheduling Cadre - The scheduling cadre interacts with program managers, engineering support teams, and the science teams to determine the day-by-day plans. Based on the plan, the cadre submits task sequences to the scheduling engine which adds them to the schedule. The scheduling engine accepts remote requests into a queue of sequences to be scheduled. The engine combines the task models with the equipment mode models to create a complete model including all timing, resources, relationships and conditions, and it then schedules the request. The scheduling cadre can use a timeline editor (mixed initiative scheduling) to make final tweaks to the schedule. The modeling and scheduling engine are powerful enough that mixed initiative scheduling will be required only in rare circumstances.

- Crew - The crew can send messages, receive messages, and read the minutes of the various planning group meetings. The crew has first-hand knowledge of the in-space situation. The crew can collaborate on the development of the preliminary schedule because they can view the task models, the equipment mode models, the developing (partial) schedule and the crew can submit scheduling requests to the instance of the scheduling engine currently being used by the ground support teams.

In addition to developing the preliminary schedule, the collaborators also develop a task list of sequences which are candidates for adding to the timeline. These may be purely discretionary or they may be sequences that are planned for the near future.

\section{B. Collaboration on Final Schedule}

The final schedule is developed in space. On the day or evening before execution, the preliminary schedule and all supporting data are transferred to an instance of the scheduling system which is co-located with the crew. The crew has immediate and full access to all features of the system and they are the main contributors to the finalization of the schedule. They have first-hand knowledge of the in-space systems; they know their own preferences and needs. They can remove omitted tasks from the schedule so that unused resources are known by the system to be available. They can modify the equipment mode models to reflect actual in-situ configurations, and they can modify task and sequence models to reflect personal experience. They can add to the schedule by selecting items from the proposed task list and submitting them to the scheduling engine so that the tasks are assigned times and so that the resource usage is tracked ${ }^{\ddagger}$. The modification to the schedule includes not only tasks done by the crew but also unattended tasks done by automated or autonomous systems such as robots. In rare circumstances the crew could use mixed-initiative scheduling to modify the timeline; however, mixed-initiative scheduling can consume a lot of valuable crew time.

Ground support teams collaborate on the final adjustments to the schedule by reviewing modifications to the models or by updating the models (updating the earth-based instance and uplinking it). They collaborate on timeline additions by reviewing what the crew adds and commenting via text messaging. The ground teams can also use remote access to submit new requests to the in-space scheduling engine.

\section{Crew Autonomy}

When a situation arises in space that necessitates modification and execution of the schedule before the ground can see the change (due to light-time delays), the crew can go ahead and make the change. It is essential for the crew

¥ On the ISS, task list items are not submitted to the scheduling engine; the crew selects them and executes them whenever they want (the crew notifies the ground of their actions). For this reason, task list items are limited to those that do not have difficult timing requirements, do not use scarce shared resources; and they are limited to tasks that are done by the crew. Additionally, the ISS crew cannot see the scheduling models of the tasks, only the procedures. 
and the in-space vehicle or habitat to be able to function if there is an extended communication loss. The crew may need to extend the timeline for a few hours or for many days. The installation of a complete planning and scheduling system in space will provide the needed capability.

\section{Collaborative Scheduling Software}

Enabling wide-spread and crew-to-earth collaboration on daily schedules as described in the preceding concept of operations requires major, but evolutionary, changes in scheduling software. In addition, a robust communication infrastructure designed for long round-trip communication delays is needed. Current development efforts are underway on delay-tolerant networking, internet in space, and publish/subscribe methods. The appendix describes interplanetary networks and the message bus architectures. Other communication infrastructure elements are planned. Relay satellites will orbit Mars to provide communication for the 12 hours per day during which the habitat will be on the far side. Additionally, a relay satellite in orbit around the sun will provide communication when the sun is directly between the earth and Mars (in worst cases, the sun-occultation communication blockage could be two weeks long).

The salient features of a scheduling system which can support the collaboration-based concept of operations are a comprehensive modeling schema that represents all the constraints, an automatic scheduler that understands the models and produces a desired schedule, remote access to the scheduling system, a human interface that is user friendly to all users including the crew, and the ability to perform over a delay-tolerant network. Figure 3 shows the components of such a scheduling system and how they are used by the collaborators. There would be two instances of the scheduling system, one on earth and another in space. The earth-based instance supports wide-spread collaboration on the preliminary schedule - the ground support teams being the primary contributors and the crew offering limited contributions. The in-space instance supports collaboration on the final schedule with the crew being the primary contributors and the ground support offering limited contributions.

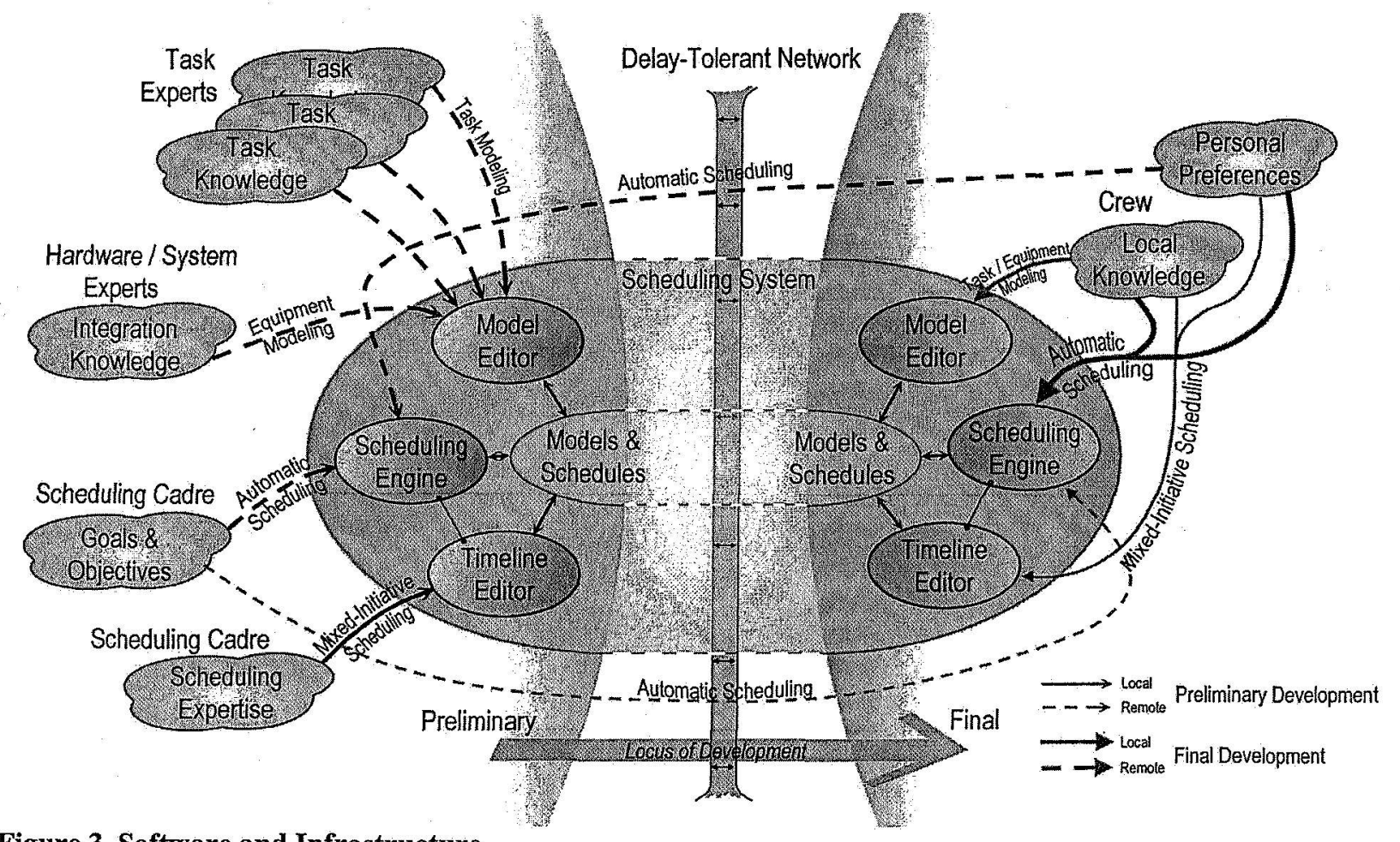

Figure 3. Software and Infrastructure

The crew will have little time to work on the schedule development. Most equipment models and task models will be pre-constructed before transferring them to the in-space software. Most of the schedule will be developed on earth. After the locus of development moves to space, the ground can review schedule additions and changes made by the crew when made far enough in advance of execution; but the crew doesn't have time to "discuss" the changes via text messages. Some crew changes to the schedule will be in response to the ongoing situation and cannot wait 
for the light-time delay for review and oversight (these changes can be called real-time replanning). The modeling schema and the scheduling engine must automatically produce valid schedules.

\section{A. Equipment Mode Modeling}

In space, as on earth, most tasks are accomplished using equipment. Most equipment have modes of operation: e.g., a microwave oven has defrost, reheat, and cook. The microwave oven's power requirement for each mode is predefined. On a space platform, the characteristics of each piece of equipment are well-known to those building and integrating the equipment into the platform systems. The equipment and their modes may be modeled independently of the tasks that will use the equipment. Equipment mode models may use multiple resources and may use other equipment in specified modes. Equipment mode models can describe a hierarchy of constraints and alternate resources. Equipment models allow low-level resources such as power to be hidden from the task modeler. The task modeler can only request these low-level resources by selecting equipment that uses them. Using equipment modes to model resource usage is an extension of a method previously proposed in Ref. 3.

Earth-based collaborators will build these models using a distributed-via-remote-access feature of the scheduling system to build models in a central data repository. Using a record locking or record checkout method allows multiple users to work at the same time as long as they work on different equipment models; revision control is built into the system. All collaborators can view the data of other collaborators.

The in-space crew can view the models in the earth-based repository via the delay-tolerant network. After the data is transferred to the in-space instance, the crew, using their knowledge of the local configurations, can make needed modifications to the models. The model viewing and editing software must be usable by the crew. The earthbased support teams can review the crew's changes to the models by either remote display or by transferring a copy of the datasets back to earth.

\section{B. Task Modeling}

A specification of the types of goals, states, activities, equipment (resources) and constraints necessary to achieve an objective is called a task model. Task models can range from simple to complex and can depend heavily on the capabilities of the scheduling system that will use them. For the most part, earth-based task experts will construct, review, and test the models before flight. The modeling schema needs to represent all the requirements so that automatic scheduling can be employed; it also needs have a straightforward representation so that the task experts (technicians, scientists, etc.) can easy enter the models; and it needs to be easy to use so that all collaborators, including the crew can understand the models. A possible modeling schema has been previously proposed (Ref. 4); some of the key features are:

- Decomposition of the operations into salient components - Operations are decomposed into tasks that define resource requirements and sequences that define relationships between tasks. Sequences can also contain other sequences, repeated tasks and sequences, and optional tasks and sequences.

- Rich expression of the relationships between components - Common-sense representations of temporal relationships using everyday concepts like sequential, during, and overlap. Innovative enhancements to represent the continuance of resource usage between tasks, the interruption of tasks, minimal percent coverage, and temporal relationships to outside tasks have been added to the modeling schema.

- Public services - The modeling schema also includes the concept of public services - models that are scheduled at the request of another model.

- Flexibility and nuances - Variable timing, alternate resources and sequences, optional items, and other nuances are modeled.

The collaborators use the same type distributed-via-remote-access features as the equipment model builders to access a central data repository. Normally, all collaborators can view the data of other collaborators; however, models containing sensitive information may be hidden to some collaborators, but never to the crew. The builders of task models can submit their models to the scheduling engine to test the models for validity and usability. Depending on the concept of operations, the results of scheduling can be part of the developing schedule or they can be tested only.

Like equipment models, the in-space crew can also view the task models via the delay-tolerant network. After the data is transferred to the in-space instance, the crew, based on their local knowledge or based on their personal preferences, can modify the task models. Complex syntactical languages, difficult to navigate user interfaces, or a modeling schema that doesn't match the real world could render such a system nearly useless to the crew. The earthbased support teams can review the crew's changes to the models by either remote display or by transferring a copy of the datasets back to earth. 


\section{Automatic Scheduling}

Automatic scheduling will be the primary mode of developing the task schedules. An incremental scheduling engine has characteristics which enable supporting multiple collaborators simultaneously contributing to the development of one schedule. An incremental engine is fed by a queue of scheduling requests (sequences of tasks). The engine adds each scheduling request to the schedule without adjusting the times of already-scheduled tasks and without introducing constraint violations or resource overbooking. The core logic of an incremental engine is usually an implementation of a greedy algorithm (Ref. 5); that is, it makes choices based only on scheduling the current request. These engines may use analytical, heuristic, algorithmic and/or artificial intelligence techniques. Incremental scheduling engine usage is depicted in Fig. 4. Some scheduling requests are emphasized to show that they may be very complex. The figure also shows that multiple queues from multiple remote users may be merged into a single queue..

As an incremental scheduling engine processes a sequence, it will search for a near-optimum schedule for the multiple tasks of the sequence being scheduled ${ }^{\S}$. The tasks always have temporal relationships to each other and may

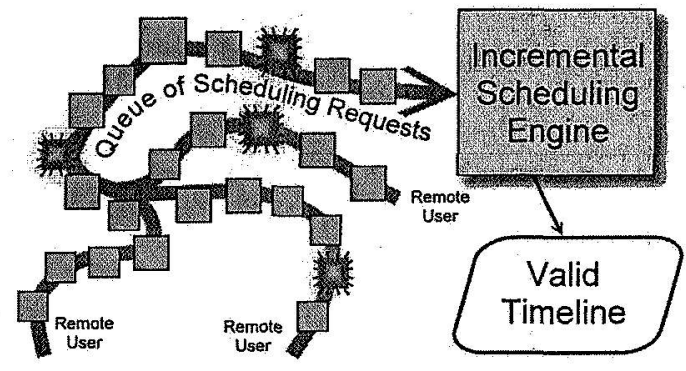

Figure 4. Incremental Engine Usage share the same resources. However, all tasks scheduled by previous scheduling requests are locked, and the residual resource profiles are treated as initial resource profiles for the current request.

Collaborators use remote access to submit scheduling requests from the repository of task models. Multiple collaborators can submit to the queue simultaneously. The scheduling system provides the ability for all users to see the current schedule as it is being developed. The crew can used the delay tolerant network to submit scheduling requests and to view the timeline.

Incremental scheduling engines and their usage is discussed in detail in Ref. 6.

\section{Mixed-Initiative Scheduling}

Mixed-initiative scheduling refers to building a timeline using a timeline editor; i.e., it is a manual process. Mixed initiative is used when the contributor knows requirements that are not described in the scheduling requests, the scheduling engine is weak, only a few new requests are to be added to the timeline, or the user wants to fully control the results (Fig. 5). Some schedule editors allow the user to move already-scheduled tasks; however, this requires the user to have global knowledge of the scheduling requirements**.

Mixed-initiative schedulers usually have logic to help the user avoid violating constraints and allow the user to override constraint limits. If the models are complete, the editor might invoke iterative-repair logic to move other tasks and eliminate constraint violation introduced by a manual edit. The editor might invoke an incremental scheduler to make slight adjustments to the user's input; this feature is called "snap-to." Additionally, the editor might use an incremental engine to suggest times where tasks can be placed without introducing constraint violations.

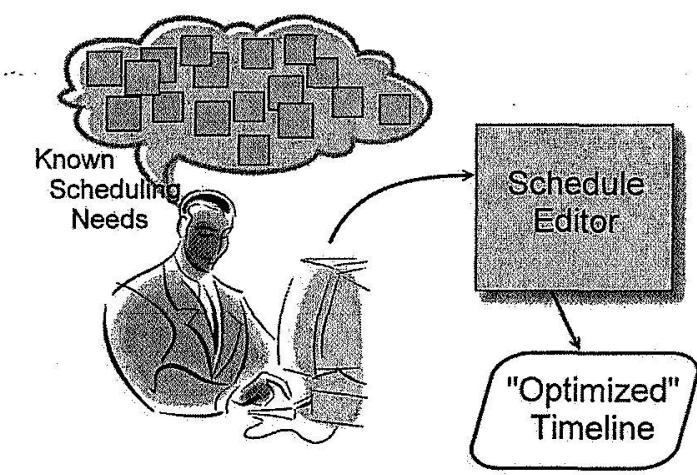

Figure 5. Mixed-Initiative Scheduling

The timeline editor will display considerable information about the schedule, the models, and the availabilities. It will be closely coupled to the data repository and the scheduling engine. It will not operate over the delay tolerant network and will be only available to local users. During the development of the preliminary schedule, a cadre of highly skilled users, called the "scheduling cadre," will be the primary users. After the locus of development moves to in-space, the crew can use mixed-initiative scheduling, but will do so rarely.

Mixed-Initiative scheduling is discussed in Ref. 6.

${ }^{8}$ Incremental engines do not provide global schedule optimization.

*** Mixed-initiative scheduling does not automatically provide global schedule optimization. However if the user is an expert and the problem is straight-forward, global optimization might be achieved. 


\section{Conclusion}

As humans explore regions of space where the earth appears as a mere point of light, and round trip communication delays exceed half an hour, it will be imperative that the humans on the journey exercise significant control over their daily schedule and the schedule of their companion systems. Yet the crew doesn't have the time to do all the scheduling. Scheduling will be a collaborative effort between multiple ground support teams and the crew.

A new concept of operations calls for developing the preliminary schedule on earth, with the ground teams being the primary contributors and the crew providing only minimal input. Once the preliminary schedule is uplinked to the in-space location, the crew will become the primary contributors and the ground teams will provide only minimal input. The concept is tailored to function effectively in the delayed communication environment.

Planning and scheduling software will evolve to meet the needs of the new concept of operations. Automatic scheduling will become the normal way to produce the schedule. Modeling will be partitioned into equipment modeling and task modeling so that different experts with different knowledge can contribute effectively. Equipment models will reflect the different modes in which the equipment operates and will book all resource and condition requirements. Task models will be grouped into sequences showing the temporal relationships of the tasks. An incremental approach to scheduling will enable multiple experts to schedule sequences without impacting sequences scheduled by other experts (the crew is among these experts). Because the models reflect all the requirements and the scheduling engine can produce good schedules, mixed-initiative scheduling will be used rarely.

The synergy of the new concept of operations, delay-tolerant communication and software, and advances in scheduling software will allow the in-space crew and earth-based support teams to collaborate on the scheduling of the tasks done by the crew, the operation of the vehicle or habitat, and the operation of the various near-autonomous robots and rovers.

\section{Appendix}

Planning and scheduling is only one application that will depend heavily on a communications framework that will carry data between Earth and Mars or in between. Without such a framework, in-space collaborative scheduling would be impossible. The cost of spaceflight being prohibitive as it is, much work will be done to leverage existing technologies and commercial hardware and software to use them and manipulate them to work in these foreign environments. Accomplishing this will necessitate standardizing communication between applications using concepts like a message bus which travels over ubiquitous protocols such as the internet modified for light-time delay.

\section{A. Inter-Planetary Internet}

Standardized digital communication devices have revolutionized the way we communicate and interact with information. But the foundation of the technology, TCP/IP, does not translate well in a space environment where line-of-sight connectivity is intermittent, communications have delays and data may have high error rates requiring retransmissions. However, the technology of TCP/IP can easily be leveraged to create Mars-based internet that connects remotely to today's Earth-based internet. The interoperability between the networks is where new technology is needed. Based on on-going research, a new concept, Delay-Tolerant Network (DTN), is evolving to mitigate the delay problem. DTN could employ concepts such as store-and-forward message delivery. In store-andforward, relay systems capture incoming data and store it

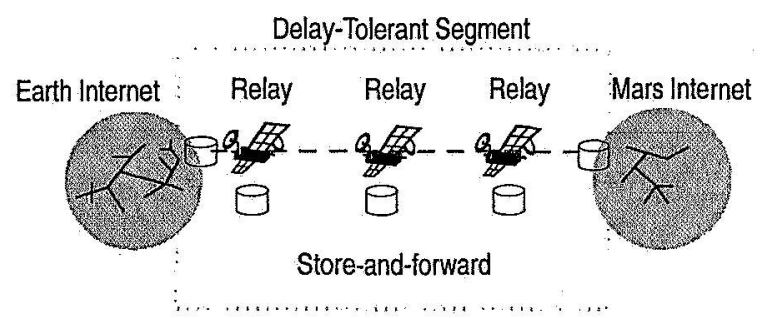

Figure 6. Inter-Planetary Internet

locally before transmitting it to the next link in a chain (Fig. 6). If there is a transfer failure, the data will not have to travel the complete distance again. DTNs can be used to route data between two traditional TCP/IP networks almost transparently to the TCP/IP packets concept. However, many of today's applications are not designed with such communication delays in mind. Loss of continual heartbeats or continuous data streams would cause many of today's applications to fail as they wait for the next packet to arrive. Therefore, any applications themselves must be built with data delays in mind. The applications must be more robust and fault tolerant and should not malfunction when there is a long data delay. 


\section{B. Message Bus}

Message buses standardize information exchange as well as data pathways between two end points. Buses can be layered upon DTNs to provide more abstraction between the software and the communication infrastructure allowing for the flexibility of architecture upgrades. Most message bus modes include a publish/subscribe protocol, sometimes called "pub/sub," in addition to more direct request/reply connections.

In the publish/subscribe mode, client applications subscribe to information they would like to be provided. As messages pass by, messages that match the requested type are captured by the client API and passed to the application.

\section{References}

${ }^{1}$ Compton, W.D., and Benson, C.D., Living and Working in Space: A History of Skylab, National Aeronautics and Space Administration, Washington, D.C., 1983.

${ }^{2} J a a p$, J.; \& Muery, K.; "Putting ROSE to Work: A Proposed Application of a Request-Oriented Scheduling Engine for Space Station Operations," Sixth International Conference on Space Operations (SpaceOps 2000), Toulouse, France, June 2000.

${ }^{3}$ Hagopian, J., and Maxwell, T., "Explicit and Implicit Resources: A Simplified Approach to User Requirements Modeling," SpaceOps 96, Fourth International Symposium on Space Mission Operations and Ground Data Systems, September 1996.

${ }^{4} J a a p$, J., Davis, E., and Richardson, L., "Maximally Expressive Modeling," Fourth International Workshop on Planning and Scheduling for Space, Darmstadt, Germany, June, 2004.

${ }^{5}$ Cormen, T.H.; Leiserson, C.E.; Rivest, R.L.; and Stein, C.; Introduction to Algorithms, 2nd Edition, Sec. 16, The MIT Press, Cambridge, Massachusetts, 2001.

${ }^{6}$ Jaap, J., and Phillips, S., "On Using an Incremental Scheduler for Human Exploration Task Scheduling," 2006 IEEE Aerospace Conference, Big Sky Montana, March 2005. 


\section{In-Space Crew-Collaborative Task Scheduling}

\section{John Jaap}

Mission Operations Laboratory

1-256-544-2226

John.Jaap@nasa.gov

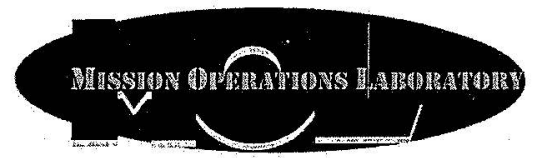

\section{Presentation Outline}

- Introduction

- Light time delays

- Interplanetary Internet

- Message Bus

- Collaboration defined

- Integration

- Concept of Operations

- Wide-spread Collaboration

- Collaboration on Final Schedule

- Collaborative Scheduling Software

- Equipment Mode Modeling

- Task Modeling

- Automatic Scheduling

- Mixed-Initiative Scheduling

- Resources, Conditions, and Autonomous Systems

- Terminology and Standards

- User Interfaces

- Conclusion 


\section{Introduction}

NASA has a vision to send humans to the moon and Mars.

These missions are long and stressful.

$\downarrow 18$ months + 4 months + return time

- The earth is a point of light $\quad$ No voice conversations with earth

The astronauts need to control their own schedules.

- Meet personal preferences Understand reasons for task times

- Have a sense of control over their own actions

- The astronauts need autonomy.

- Quick response to anomalies

- Extended loss of communication

Technological advances are available to help

- Delay-tolerant networks

- Remote-access planning and scheduling systems

In-Space Crew-Collaborative

Task Scheduling

19. June, 2006

\section{Light Time Delays,(to Mars)}

- Close Approach is $56 \times 10^{6} \mathrm{~km}$ or 3.1 minutes (one way)

- Far Retreat is $400 \times 10^{6} \mathrm{~km}$ or 22.2 minutes (one way)

- The earth overtakes M every 26 months.

Relay satellites can he

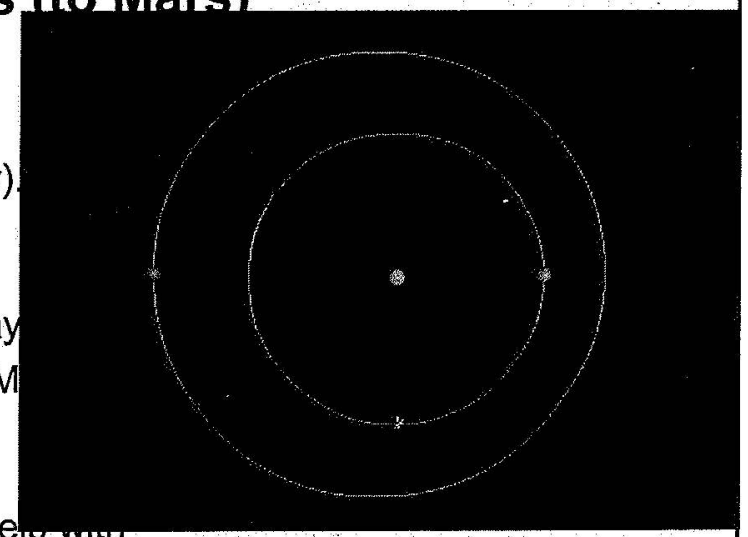
solar occultation and the delay-tolerant network.

Diagram shows a relay satellite trailing the earth by 90 degrees 


\section{Communications for Mars Exploration}

Interplanetary Internet

- Path known even when destination is unavailable.

- Long time delays expected.

- Store-and-forward is a good approach

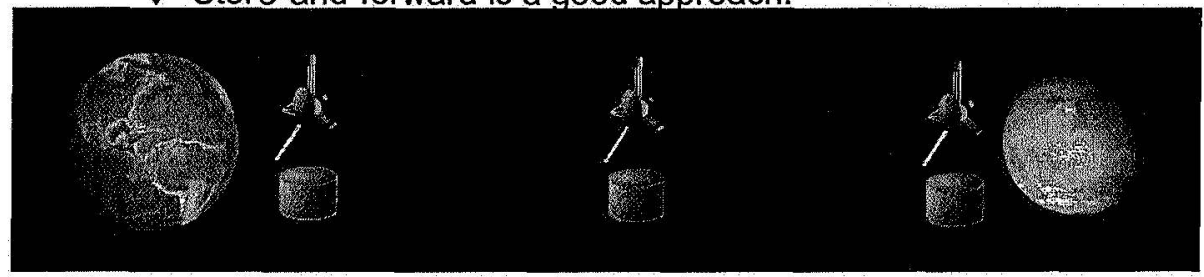

Message Bus

- Publish / subscribe (one-way communication)

- Standard infrastructure, standard message protocol

In-Space Crew-Collaborative
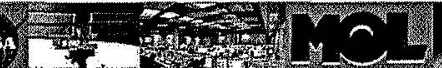

\section{Collaboration in General}

Interactive Collaboration

$\rightarrow$

$\rightarrow$ Teteconfereneing and webeonfereneing

- Custom software "Tr'

$\rightarrow$ Text Messaging

- File transfer

- Electronic forums

- Electronic mail

$\rightarrow$ Postatmail

- Passive Collaboration

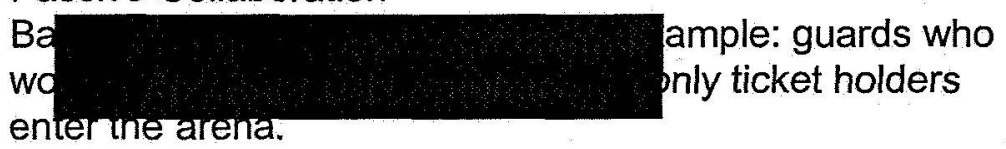

In-Space Crew-Collaborative 


\section{Concept of Operations}

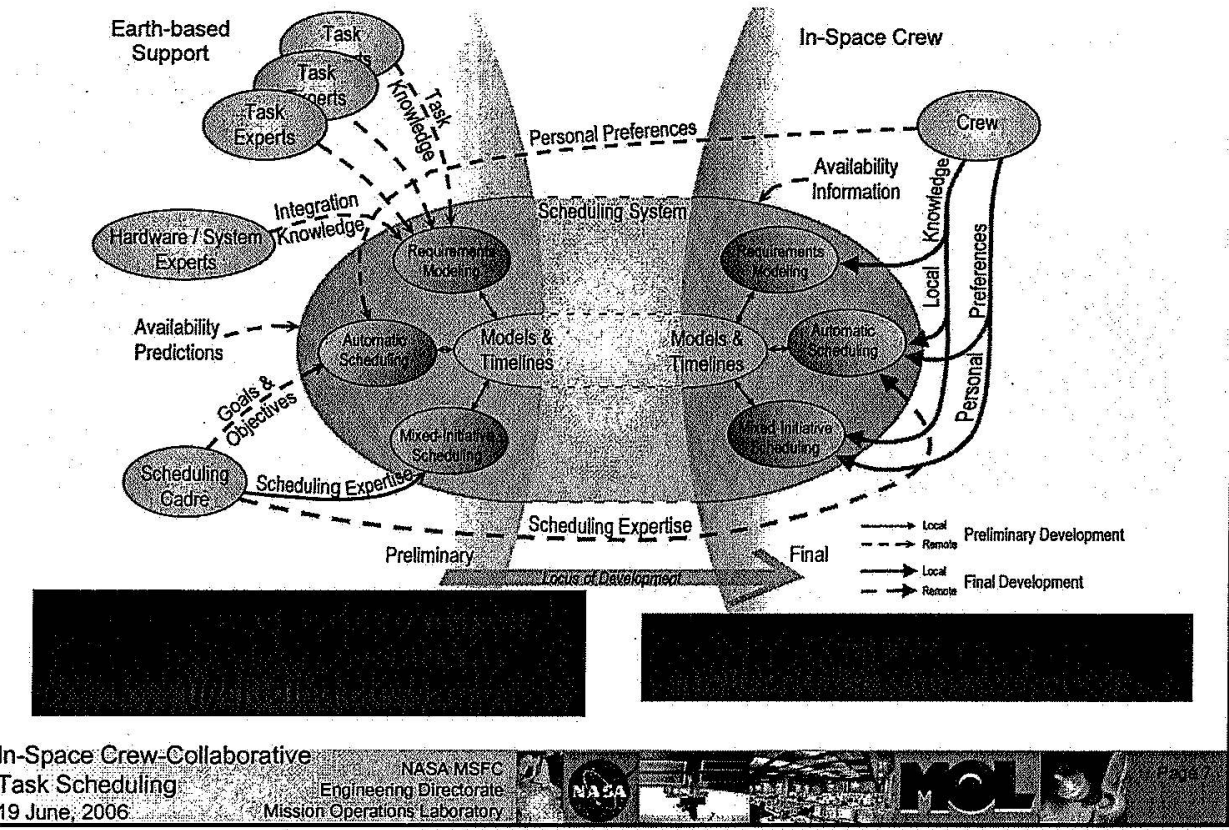

\section{Contributors to Wide-Spread Collaboration}

on Preliminary Timeline

- Task Experts -

First-hand knowledge about the tasks to be done and how to order the tasks to accomplish the goals.

- Hardware and Systems Experts -

Detailed knowledge about how the hardware performs and how it is integrated with systems.

- Scheduling Cadre -

Knowledge of program goals; produce the detailed timelines. Final tweaks to timeline.

- Crew -

First-hand knowledge of in-space situation, personal preferences.

- Other Contributors -

Provide availabilities, predictions, and simulation of 


\section{Contributors to Final Timeline}

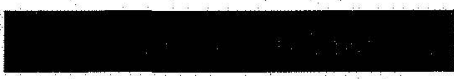

$\checkmark$ Crew -

Modify timeline based on in-space situation, personal preferences, etc.

Modify models based on actual configurations.

Delete tasks as desired / needed.

Add tasks as desired / needed.

- Scheduling Cadre -

Verify actions of crew.

Modify models and timeline as needed.
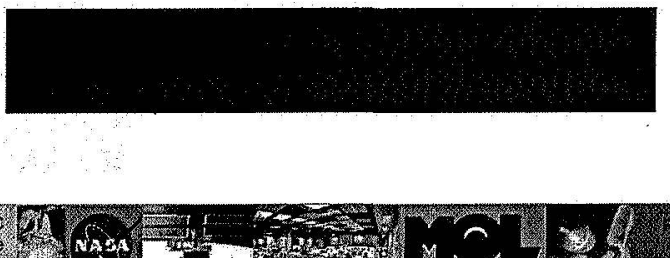

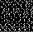




\section{P\&S Software - Modeling}

- Equipment Mode Modeling

- Equipment and their modes are modeled independently of the tasks that use the equipment.

- Resource and condition constraints are assigned in the equipment modes.

- Models can define a hierarchy of constraints and alternate constraints.

- Task Network Modeling

- Tasks use equipment in specified modes, variable durations.

- Temporal networks of tasks are defined using relationships like during, after, overlap, cyclic, etc.

- Variable timing.

- Optional tasks.

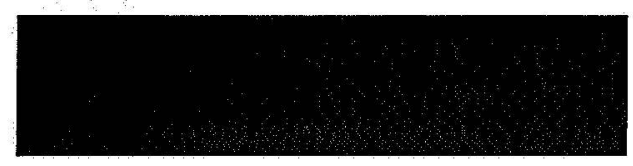

In-Space Crew-Collaborative:w;

Task Scheduling , \%

\section{P\&S Software - Scheduling}

- Automatic Scheduling

- An incremental scheduling engine can support collaboration as defined by the concept of operations.

\section{Mixed-Initiative Scheduling}

- Manual process best used by scheduling experts only to fine tune timeline.

- Can be used to delete tasks.

- Can be used to invoke incremental engine to add to timeline.
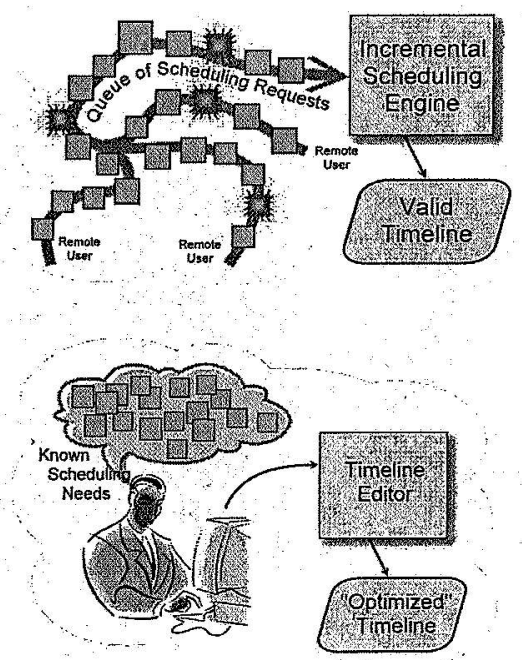

In-Space Crew-Collaborative 


\section{P\&S Software - Resources, Conditions, and Autonomous Systems}

- Resources

(power, camera, storage lockers)

- Conditions

(sunlight, communications, weather)

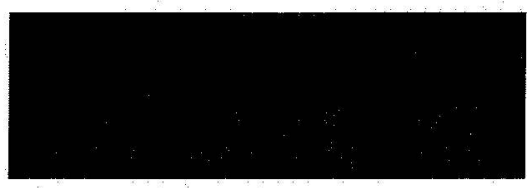

- Autonomous Systems (robots, rovers, etc.)
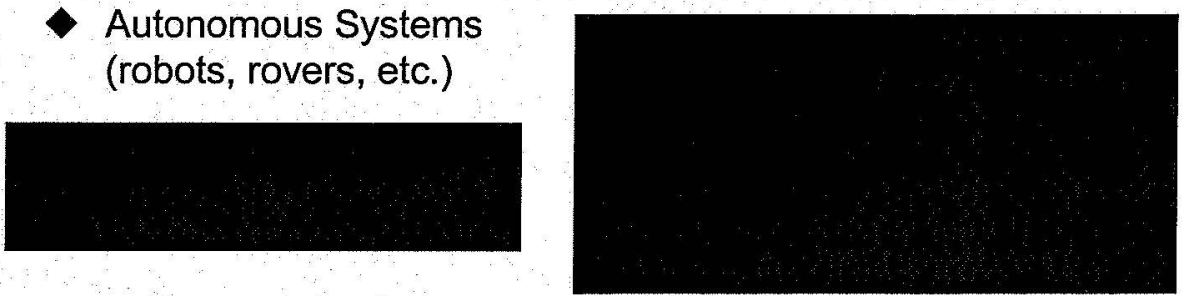

In-Space Creweollaborativew

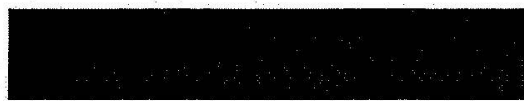

\section{P\&S Software - Terminology and Standards}

- Collaboration by many diverse contributors requires standard terminology and standard methods.

- Crew collaboration and crew autonomy require standard terminology and software.

- Current / Historic scheduling community is fragmented. Examples:

- Terminology:

+ State vs. condition.
- Approach:

+ Start and end events vs. task duration.

+ Implicit resource usage.

In-Space Crew-Collaborative 


\section{P\&S Software - User Interfaces}

Collaboration requires good user interfaces.

- Experts in many diverse fields must become "virtual" scheduling experts.

- Scheduling experts must be able to comprehend the requirements entered by others.

$\checkmark$ The Crew must be able to use any part.

The Crew will need specialized user interfaces.
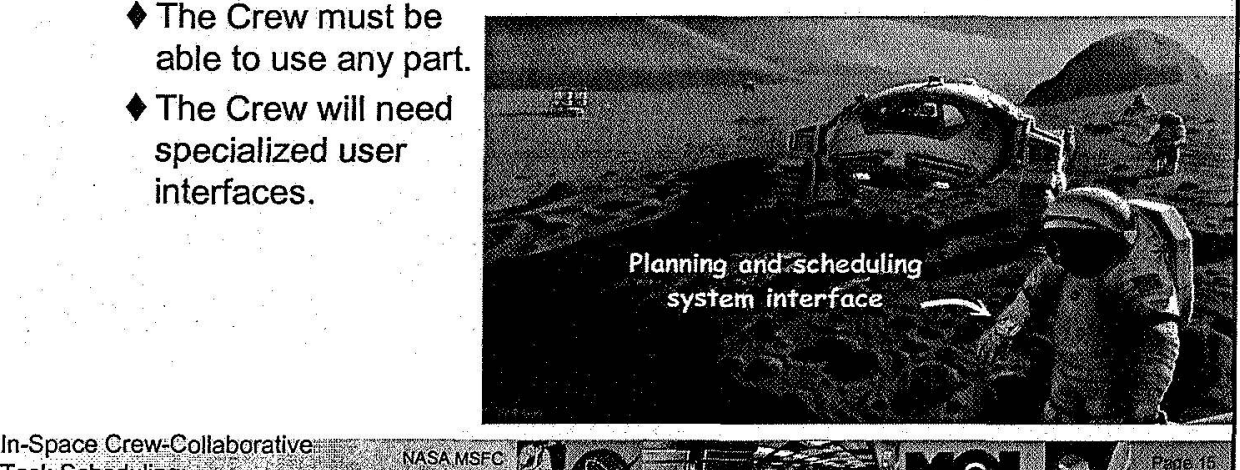

\section{Conclusion}
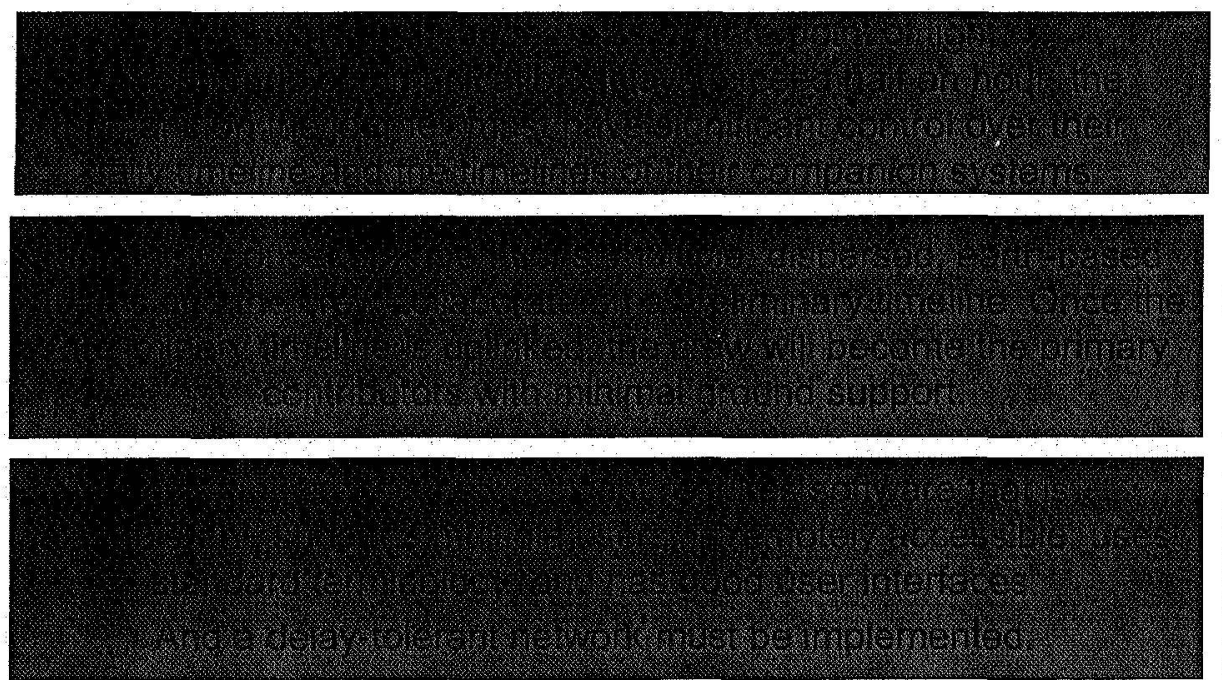

In-Space Grew-Collaborative

Task Scheduling NASA MSEV 19 June, 2006 